\title{
On The Distance Between
}

\section{Distinct Group Latin Squares}

\section{Diane Donovan}

Centre for Combinatorics, Department of Mathematics, The University of Queensland, Qld, 4072, Australia.

\section{Sheila Oates-Williams}

Centre for Combinatorics, Department of Mathematics, The University of Queensland, Qld, 4072, Australia.

Cheryl E. Praeger ${ }^{1}$

Department of Mathematics, The University of Western Australia, Nedlands, WA, 6907, Australia.

\section{ABSTRACT}

In a paper in 1992, Drápal addressed the question of how far apart the multiplication tables of two groups can be? In this paper we continue this investigation; in particular we study the interaction between partial equalities in the multiplication tables of the two groups and their subgroup structure.

\footnotetext{
${ }^{1}$ This work was undertaken while the third author held a Raybould Visiting Fellowship at the University of Queensland
} 


\section{Introduction}

In 1958 Fuchs [4] posed two problems about the distance between the multiplication tables of two groups.

In order to explain these questions we define a Cayley matrix.

DEFINITION 1.1 A matrix $\left(a_{i j}\right)$, where $\left\{a_{i j} \mid 1 \leq i, j \leq n\right\}=\{1, \ldots, n\}$ is a Cayley matrix if it is a Latin square which satisfies the quadrangle criterion; that is, for all indices $i, i_{1}, j, j_{1}, k, k_{1}, l, l_{1}$, it follows from the equations $a_{j k}=a_{j_{1} k_{1}}, a_{i k}=a_{i_{1} k_{1}}$, $a_{i l}=a_{i_{1} l_{1}}$ that $a_{j l}=a_{j_{1} l_{1}}$.

Such a matrix can be associated with a group by choosing an arbitrary row and an arbitrary column to correspond to the identity of the group and then using these as the headline and sideline to form a multiplication table.

Using this definition we can reword Fuchs' problems as follows.

For a positive integer $n$, determine the largest integers $k_{1}(n)$ and $k_{2}(n)$ such that, for all groups $G$ of order $n$,

(a) if $k_{1}(n)$ elements are deleted at random from a Cayley matrix of $G$, then the rest of the Cayley matrix determines $G$ up to isomorphism, and

(b) if $k_{2}(n)$ elements are deleted at random from a Cayley matrix of $G$, then the Cayley matrix can be reconstructed uniquely from its remaining part.

Since the deletion of two rows (or two columns) of a Cayley matrix would allow their interchange in any reconstruction, $k_{2}(n) \leq 2 n-1$. Dénes [2, Lemmas 2 and 3 and 
Theorem 1] proved that for $n \neq 4,6, k_{2}(n)=2 n-1$, (and is independent of the group). He also [2, Theorem 2] showed that $k_{1}(4)=k_{2}(4)=3$. Dénes's results could also be regarded as saying that the minimum distance between two Cayley matrices of order $n$ is $2 n$.

Drápal [3] considered a slightly more restrictive case, where the matrix is taken to be a multiplication table of a group with a fixed headline and sideline. He [3, Theorem 6.2] showed that the minimal distance between the multiplication tables of groups $(G, \circ)$ and $(G, *)$, of order $n \geq 51$, is $\delta_{0}((G, \circ))$, where $\delta_{0}((G, \circ))$ is $6 n-18$ if $n$ is odd, $6 n-20$ if $(G, \circ)$ is dihedral of twice odd order $n$, and $6 n-24$ in all other cases. Moreover, he showed that for each $(G, \circ)$ there exists a group $(G, *)$ such that the two multiplication tables differ in exactly $\delta_{0}((G, \circ))$ entries. Drápal also showed [3, Theorem 6.2] that the multiplication tables of non-isomorphic groups must differ in at least $n^{2} / 9$ entries. He conjectured that since there are no known examples of non-isomorphic groups of order $n$ with multiplication tables which differ in less than $n^{2} / 4$ entries, that this bound may be improved to $n^{2} / 4$.

The aim of this paper is to investigate the influence that the subgroup structure of a group has on the structure of its multiplication table.

To be more precise, suppose that $(G, \circ)$ and $(G, *)$ are distinct groups based on the same carrier set $G$ of order $n$, say. Choose two (not necessarily distinct) orderings of the elements of $G$ and consider the multiplication tables $L$ and $L^{*}$ for $(G, \circ)$ and $(G, *)$ respectively, obtained by using the elements of $G$ in the first ordering on the headlines of both $L$ and $L^{*}$ and in the second ordering on the sidelines of both $L$ and $L^{*}$. Thus $L$ and $L^{*}$ are $n \times n$ arrays with rows and columns indexed by $G$ (in the 
chosen orderings) such that, for $g, g^{\prime} \in G$, the $\left(g, g^{\prime}\right)$-entry is $g \circ g^{\prime}$ in $L$ and $g * g^{\prime}$ in $L^{*}$. We shall consider certain square subarrays of order $m$ of the multiplication tables $L$ and $L^{*}$, by which we mean subarrays indexed by some $m$-element subsets of the rows and columns (the rows and columns may be indexed by different subsets of $G$ ).

In Section 2 we assume equality of certain collections of entries of $L$ and $L^{*}$ and conduct a preliminary study of the influence of the subgroup structure of $(G, \circ)$ on the subgroup structure of $(G, *)$. We begin this section with a result (Lemma 2.2) that shows that, if $(H, \circ)$ is a subgroup of $(G, \circ)$ of index $r$, and if $L$ and $L^{*}$ agree on a subarray indexed by the rows of $H$ and the columns of some right coset $H \circ x$ (or on a subarray indexed by the rows of some left coset $y \circ H$ and the columns of $H$ ) then $(H, *)$ is a subgroup of $(G, *)$ and the multiplication tables of $(H, \circ)$ and $(H, *)$ are identical. We also show (Theorem 2.5) that, under certain conditions, if $L$ and $L^{*}$ agree on two particular subarrays and in one entry of a certain third subarray, then they agree in every entry of this subarray. This section also contains two results (Corollary 2.3 and Lemma 2.4) concerning the number of equal entries of $L$ and $L^{*}$ under certain extra conditions.

In Section 3 we continue our investigation of the relations between the subgroup structures of $(G, \circ)$ and $(G, *)$. We assume that the conclusions of Lemma 2.2 hold so that $H$ is a subgroup of both $(G, \circ)$ and $(G, *)$ such that the multiplication tables of $(H, \circ)$ and $(H, *)$ are identical. We assume in addition that, for some $x, y \in G$, $y \circ H \circ x=y * H * x$ (as sets), thus determining a bijection $\phi_{y x}: H \rightarrow H$ defined by $y \circ h \circ x=y *(h) \phi_{y x} * x$ for $h \in H$. First we show (Theorem 3.3) that $L$ and $L^{*}$ agree on a subarray indexed by the rows of some left coset $y \circ H$ and the columns of some 
right coset $H \circ x$ if and only if $\phi_{y x}$ is defined and $\phi_{y x}=\phi_{1 x}=\phi_{y 1}$ is an isomorphism from $(H, \circ)$ to $(H, *)$, and moreover if either $x$ or $y$ lies in $H$ then $\phi_{y x}$ is the identity map.

There are many other questions one could ask about the relationship between properties which are common to the two groups $(G, \circ)$ and $(G, *)$ and equalities of entries of certain subarrays of the multiplication tables $L$ and $L^{*}$. Some of these are posed and discussed in Section 4.

\section{Group Identities and subgroups}

Let $(G, \circ)$ and $(G, *)$ be groups of order $n$, and let $\mathcal{L}^{\circ}$ and $\mathcal{L}^{*}$ be the corresponding collections of multiplication tables, obtained from all possible reorderings of the rows and columns of given multiplication tables for $(G, \circ)$ and $(G, *)$, respectively.

Since we assume that the carrier set $G$ is the same for the two groups, the set of rows, and the set of columns, of any element of $\mathcal{L}^{\circ}$ or $\mathcal{L}^{*}$ will be indexed by $G$. Thus, for $g, g^{\prime} \in G$, we shall speak of the entry in row $g$ and column $g^{\prime}$ in one of these squares as its $\left(g, g^{\prime}\right)$-entry. We shall call $L^{*} \in \mathcal{L}^{*}$ the element of $\mathcal{L}^{*}$ corresponding to $L \in \mathcal{L}^{\circ}$ if $L^{*}$ has the same headline and sideline as $L$. We shall specify the orderings of the rows or columns only when it will be helpful for the discussion. It will be helpful to have such an order specified when we wish to focus on, say, some subgroup $(H, \circ)$ of $(G, \circ)$ and its effect on the structure of squares in $\mathcal{L}^{\circ}$.

DEFINITION 2.1 Suppose that $(H, \circ)$ is a subgroup of $(G, \circ)$ of index $r$. We define a canonical element $L(G, \circ, H)$ of $\mathcal{L}^{\circ}$ corresponding to $H$ as follows. 
(i) Choose an ordering of $H$, say $h_{1}=e, h_{2}, \ldots, h_{m}$, where $e$ is the identity of $(G, \circ)$, and $n=m r$.

(ii) Choose orderings of the right cosets and the left cosets of $H$ in $G$; $H \circ x_{1}, H \circ x_{2}, \ldots, H \circ x_{r}$, and $\quad y_{1} \circ H, y_{2} \circ H, \ldots, y_{r} \circ H$, where $x_{1}=y_{1}=e$, and set $\mathbf{x}=\left(x_{1}, \ldots, x_{r}\right)$ and $\mathbf{y}=\left(y_{1}, \ldots, y_{r}\right)$.

(iii) Define $L(G, \circ, H)$ to be the multiplication table of $G$ with respect to indexing the rows by $y_{1} \circ h_{1}, \ldots, y_{1} \circ h_{m}, y_{2} \circ h_{1}, \ldots, y_{r} \circ h_{m}$, and the columns by $h_{1} \circ x_{1}, \ldots, h_{m} \circ x_{1}, h_{1} \circ x_{2}, \ldots, h_{m} \circ x_{r}$.

Let $L(H)$ denote the multiplication table of $(H, \circ)$ with respect to the ordering of $(\mathrm{i})$. Then the rows of $L(G, \circ, H)$ are partitioned according to the partition of $G$ into left cosets of $H$, and the columns of $L(G, \circ, H)$ are partitioned according to the partition of $G$ into right cosets of $H$; the square subarray, which is also a Latin subsquare, corresponding to the rows of $y_{i} \circ H$ and the columns of $H \circ x_{j}$ is $y_{i} \circ L(H) \circ x_{j}$. We shall call this decomposition of $L(G, \circ, H)$ into the subsquares $y_{i} \circ H \circ x_{j}$, where $1 \leq i, j \leq r$, the canonical coset decomposition corresponding to $H$. It depends on the orderings chosen in Definition 2.1 (i) and (ii).

It is obvious that if $e, e_{*}$ are the identities of $(G, \circ)$ and $(G, *)$ respectively, then either $e=e_{*}$, or for all $g \in G, g \neq e * g, g * e, e_{*} \circ g$ or $g \circ e_{*}$.

LEMMA 2.2 Let $(H, \circ)$ be a subgroup of $(G, \circ)$ of index $r>1$, and suppose that the element $L^{*} \in \mathcal{L}^{*}$ corresponding to $L(G, \circ, H)$ agrees with $L(G, \circ, H)$ in at least one of the Latin subsquares of the form $y_{i} \circ L(H)$ or $L(H) \circ x_{i}$, for some $1 \leq i \leq r$. 
Then $e=e_{*},(H, *)$ is a subgroup of $(G, *)$, and moreover the multiplication tables of $(H, \circ)$ and $(H, *)$ are identical.

Proof. Suppose that there is a subsquare $L(H) \circ x_{j}$ which agrees everywhere with the corresponding rows and columns of $L^{*}$. (The proof in the other case is similar.) Then by the preceeding remark it follows that $e=e_{*}$. Also, for all $h, h^{\prime} \in H$, the $\left(h, h^{\prime} \circ x_{j}\right)$ entries in the two multiplication tables are equal by assumption, so we have $h *\left(h^{\prime} \circ x_{j}\right)=h \circ\left(h^{\prime} \circ x_{j}\right)$, and in particular $h * x_{j}=h \circ x_{j}$ for all $h \in H$. Thus we have that for all $h, h^{\prime} \in H:\left(h \circ h^{\prime}\right) * x_{j}=\left(h \circ h^{\prime}\right) \circ x_{j}$ which by associativity equals $h \circ\left(h^{\prime} \circ x_{j}\right)=h *\left(h^{\prime} \circ x_{j}\right)=h *\left(h^{\prime} * x_{j}\right)$ which by associativity (of $*$ this time) equals $\left(h * h^{\prime}\right) * x_{j}$. Then, multiplying in $(G, *)$ on the right by the inverse $x_{j}^{*}$ of $x_{j}$, we obtain $h \circ h^{\prime}=h * h^{\prime}$. It follows that $(H, *)$ is a subgroup of $(G, *)$ which has identical multiplication table to that of $(H, \circ)$.

COROLLARY 2.3 Let $(H, \circ)$ be a subgroup of $(G, \circ)$ of index $r>1$, and suppose that the element $L^{*} \in \mathcal{L}^{*}$ corresponding to $L(G, \circ, H)$ agrees with $L(G, \circ, H)$ in at least $r^{2}-2 r+2$ of the $r^{2}$ Latin subsquares of the canonical coset decomposition corresponding to $H$. Then $e=e_{*},(H, *)$ is a subgroup of $(G, *)$ and the multiplication tables of $(H, \circ)$ and $(H, *)$ are identical.

Proof. Since at least $r^{2}-2 r+2$ of the subsquares of $L(G, \circ, H)$ agree everywhere with $L^{*}$, there is at least one subsquare of the form $y_{i} \circ L(H)$ or $L(H) \circ x_{j}$ which agrees everywhere with the corresponding rows and columns of $L^{*}$. The result now follows from Lemma 2.2 . 
LEMMA 2.4 Let $(H, \circ)$ be a subgroup of $(G, \circ)$ of order $m$, and suppose that the element $L^{*} \in \mathcal{L}^{*}$ corresponding to $L(G, \circ, H)$ agrees with $L(G, \circ, H)$ in at least the Latin subsquares of the form $y \circ L(H)$ and $L(H) \circ x$, for some $x, y \in G$. Then the number of entries of $L^{*}$ which agree with $L(G, \circ, H)$ in the subsquare $y \circ L(H) \circ x$ is

$$
m\left(m-\left|T_{y x}\right|\right)
$$

where $T_{y x}=\{h \in H \mid y \circ h \circ x \neq y * h * x\}$.

Proof. If $T_{y x}=\{h \in H \mid y \circ h \circ x \neq y * h * x\}$, then

$$
\mid\left\{\left(y h_{1}, h_{2} x\right) \mid h_{1} \in H, h_{2} \in H \text { and } y \circ h_{1} \circ h_{2} \circ x \neq y * h_{1} * h_{2} * x\right\} \mid
$$

equals $|H| \cdot\left|T_{y x}\right|$ and the result is immediate.

THEOREM 2.5 Let $H=\left\{e, h_{2}, \ldots, h_{m}\right\}$ be a subgroup of $(G, \circ)$ and suppose that there is an element $y \in G$ such that $y$ and $y^{-1}$ (the inverse of $y$ in $(G, \circ)$ ) are both left and right coset representatives as in Definition 2.1. Suppose in addition that

1. $y \circ H=H \circ y$,

2. $y \circ h_{s} \circ y^{-1}=y * h_{s} * y^{-1}$ for some (fixed) $h_{s} \in H$, and

3. the element $L^{*} \in \mathcal{L}^{*}$ corresponding to $L(G, \circ, H)$ agrees with $L(G, \circ, H)$ either

(a) in the Latin subsquares $L(H) \circ y^{-1}$ and $y^{-1} \circ L(H)$, or

(b) in the Latin subsquares $L(H) \circ y$ and $y \circ L(H)$.

Then $L^{*}$ and $L(G, \circ, H)$ also agree in the Latin subsquare $y \circ L(H) \circ y^{-1}$. 
Proof. We give the details of the proof for assumption 3(a). The proof for 3(b) is analogous.

By Lemma 2.2, the multiplication tables for $(H, \circ)$ and $(H, *)$ are identical. Since $y \circ H=H \circ y$ it follows that $y^{-1} \circ H=H \circ y^{-1}$ and $y \circ H \circ y^{-1}=H$.

Choose any $h_{t} \in H$.

$$
\begin{aligned}
y \circ h_{t} \circ y^{-1} & =y \circ h_{s} \circ h_{r} \circ y^{-1}, \quad \text { for some } r \\
& =y \circ h_{s} \circ y^{-1} \circ h_{p}, \quad \text { for some } p, \\
& =\left(y \circ h_{s} \circ y^{-1}\right) * h_{p}, \quad \text { since } y \circ h_{s} \circ y^{-1} \in H, \text { and }(H, \circ)=(H, *), \\
& =\left(y * h_{s} * y^{-1}\right) * h_{p}, \quad \text { by assumption } 2 .
\end{aligned}
$$

By assumption 3(a) $y^{-1} * h_{p}=y^{-1} \circ h_{p}$ and by the definition of $h_{r}$, and assumption 3(a) again $y^{-1} \circ h_{p}=h_{r} \circ y^{-1}=h_{r} * y^{-1}$. So $y^{-1} * h_{p}=h_{r} * y^{-1}$. Hence

$$
\begin{aligned}
y \circ h_{t} \circ y^{-1} & =\left(y * h_{s}\right) *\left(h_{r} * y^{-1}\right) \\
& =y *\left(h_{s} * h_{r}\right) * y^{-1} \\
& =y * h_{t} * y^{-1}
\end{aligned}
$$

(since $(H, *)=(H, \circ))$.

Now, if $y$ and $y^{-1}$ are not coset representatives, then we let $x_{i}, x_{l}, y_{j}$ and $y_{k}$ be the coset representatives, as in Definition 2.1, for $H \circ y^{-1}, H \circ y, y \circ H, y^{-1} \circ H$ respectively and proceed as follows.

COROLLARY 2.6 Let $H=\left\{e, h_{2}, \ldots, h_{m}\right\}$ be a subgroup of $(G, \circ)$ and suppose that there is an element $y \in G$ such that 
1. $y \circ H=H \circ y$,

2. $y_{j} \circ h_{s} \circ x_{i}=y_{j} * h_{s} * x_{i}$ for some (fixed) $h_{s} \in H$, and

3. the element $L^{*} \in \mathcal{L}^{*}$ corresponding to $L(G, \circ, H)$ agrees with $L(G, \circ, H)$ either

(a) in the Latin subsquares $L(H) \circ x_{i}$ and $y_{k} \circ L(H)$, or

(b) in the Latin subsquares $L(H) \circ x_{l}$ and $y_{j} \circ L(H)$.

Then $L^{*}$ and $L(G, \circ, H)$ also agree in the Latin subsquare $y_{j} \circ L(H) \circ x_{i}$.

Proof. As for Theorem 2.5, we give the proof only for assumption 3(a), that for 3(b) being analogous.

By the definition of $x_{i}$ and $y_{j}$, and assumption $1, y_{j} \circ H \circ x_{i}=y \circ H \circ y^{-1}=H$ and also $H \circ x_{i}=H \circ y^{-1}=y^{-1} \circ H=x_{i} \circ H$.

As in Theorem 2.5 we note that for any $h_{t} \in H, h_{t}=h_{s} \circ h_{r}=h_{s} * h_{r}$ for some $r$, and $h_{r} \circ x_{i}=x_{i} \circ h_{p}$ for some $p$, and we obtain

$$
y_{j} \circ h_{t} \circ x_{i}=\left(y_{j} * h_{s} * x_{i}\right) * h_{p}
$$

By our observation above, $x_{i} \circ H=y^{-1} \circ H=y_{k} \circ H$, so $x_{i}=y_{k} \circ h_{q}$ for some $q$. Using this, and assumption $3(\mathrm{a})$, and the fact that $(H, \circ)=(H, *)$, we get $x_{i} \circ h_{p}=$ $y_{k} \circ\left(h_{q} \circ h_{p}\right)=y_{k} *\left(h_{q} \circ h_{p}\right)=y_{k} *\left(h_{q} * h_{p}\right)$. Since $x_{i}=y_{k} \circ h_{q}=y_{k} * h_{q}$, we have $x_{i} \circ h_{p}=x_{i} * h_{p}$. Then, by the definition of $h_{r}$, and assumption 3(a), $x_{i} * h_{p}=x_{i} \circ h_{p}=h_{r} \circ x_{i}=h_{r} * x_{i}$. Finally we have

$$
\begin{aligned}
y_{j} \circ h_{t} \circ x_{i} & =\left(y_{j} * h_{s}\right) *\left(h_{r} * x_{i}\right) \\
& =y_{j} * h_{t} * x_{i} .
\end{aligned}
$$


COROLLARY 2.7 Let $H=\left\{e, h_{2}, \ldots, h_{m}\right\}$ be a subgroup of $(G, \circ)$ of index 2, and suppose that the element $L^{*} \in \mathcal{L}^{*}$ corresponding to $L(G, \circ, H)$ agrees with $L(G, \circ, H)$ in the Latin subsquares of the form $x \circ L(H)$ and $L(H) \circ x$, where $x \notin H$, and for some fixed $s, 1 \leq s \leq m, x \circ h_{s} \circ x=x * h_{s} * x$. Then $L^{*} \in \mathcal{L}^{*}$ agrees with $L(G, \circ, H)$ everywhere.

\section{$3 \quad$ Subgroups and bijections}

In this section we assume that $(G, \circ)$ and $(G, *)$ are groups of order $n$, and that $H$ is a subgroup of both $(G, \circ)$ and $(G, *)$ such that the multiplication tables of $(H, \circ)$ and $(H, *)$ are identical. Thus we are assuming that the conclusion of Lemma 2.2 holds. We shall say that these groups have a consistent right (left) coset decomposition with respect to $\mathbf{x}=\left(x_{1}, \ldots, x_{r}\right)$, if $H \circ x_{i}=H * x_{i}\left(x_{i} \circ H=x_{i} * H\right.$ respectively) for $i=1, \ldots, r$. Also we shall say that they have a consistent 2-sided coset decomposition with respect to $\mathbf{x}=\left(x_{1}, \ldots, x_{r}\right)$ and $\mathbf{y}=\left(y_{1}, \ldots, y_{r}\right)$ if the groups have a consistent right coset decomposition with respect to $\mathbf{x}$ and a consistent left coset decomposition with respect to $\mathbf{y}$. Under these conditions, of course, for all $g \in G, H * g=H \circ g$ and $g * H=g \circ H$. However the existence of a consistent 2-sided coset decomposition does not ensure the equality of all "2-sided" cosets $y * H * x$ and $y \circ H \circ x$, even if the multiplication tables of $(H, *)$ and $(H, \circ)$ are identical; see for example the subgroup $H_{3}$ in the example after Corollary 3.2. We shall consider $L(G, \circ, H)$ and the element $L^{*} \in \mathcal{L}^{*}$ corresponding to $L(G, \circ, H)$. ( $L^{*}$ is not, of course, in general the same as 
$L(G, *, H)$, since the cosets of $H$ in the $(G, \circ)$ and $(G, *)$ are only equal as sets.)

As in Section 2, we order the right and left cosets of $(H, \circ)$ in $G$ as $H \circ x_{1}, H \circ$ $x_{2}, \ldots, H \circ x_{r}$, and $y_{1} \circ H, y_{2} \circ H, \ldots, y_{r} \circ H$, where $x_{1}=y_{1}=e$. Under our assumption of a consistent 2-sided coset decomposition, for each $i, j$, with $1 \leq i, j \leq r$, there are bijections $\phi_{i 1}$ and $\phi_{1 j}$ on $H$ determined by the equations $y_{i} \circ h=y_{i} *(h) \phi_{i 1}$ and $h \circ x_{j}=(h) \phi_{1 j} * x_{j}$ respectively, for $h \in H$. If, further, we assume that for some $x_{j}, y_{i}$ we have $y_{i} \circ H \circ x_{j}=y_{i} * H * x_{j}$ then we have a bijection $\phi_{i j}$ on $H$ determined similarly by $y_{i} \circ h \circ x_{j}=y_{i} *(h) \phi_{i j} * x_{j}$. By our assumptions about $H, \phi_{11}$ is the identity map. Also, since $e=e^{*}$,

$$
(e) \phi_{i 1}=(e) \phi_{1 j}=e
$$

for all $i$ and $j$. We shall investigate restrictions on the maps $\phi_{i j}$ implied by certain equalities in the entries of $L(G, \circ, H)$ and $L^{*}$.

PROPOSITION 3.1 Let $h, h^{\prime} \in H$, and $1 \leq i, j \leq r$, and if both $i$ and $j$ are greater than 1, also assume that $\phi_{i j}$ is defined. Then the $\left(y_{i} \circ h, h^{\prime} \circ x_{j}\right)$-entries of $L(G, \circ, H)$ and $L^{*}$ are equal if and only if

$$
\left(h \circ h^{\prime}\right) \phi_{i j}=(h) \phi_{i 1} *\left(h^{\prime}\right) \phi_{1 j}
$$

Proof. The $\left(y_{i} \circ h, h^{\prime} \circ x_{j}\right)$-entries of $L(G, \circ, H)$ and $L^{*}$ are equal if and only if $\left(y_{i} \circ h\right) \circ\left(h^{\prime} \circ x_{j}\right)=\left(y_{i} \circ h\right) *\left(h^{\prime} \circ x_{j}\right)$, that is if and only if $y_{i} *\left(h \circ h^{\prime}\right) \phi_{i j} * x_{j}=$ $y_{i} *(h) \phi_{i 1} *\left(h^{\prime}\right) \phi_{1 j} * x_{j}$, which is true if and only if $\left(h \circ h^{\prime}\right) \phi_{i j}=(h) \phi_{i 1} *\left(h^{\prime}\right) \phi_{1 j}$.

We shall explore some consequences of this result. 
COROLLARY 3.2 Let $h \in H$, and $1 \leq i, j \leq r$, and if both $i$ and $j$ are greater than 1, assume also that $\phi_{i j}$ is defined.

(a) The $\left(y_{i} \circ h, x_{j}\right)$-entries of $L(G, \circ, H)$ and $L^{*}$ are equal if and only if

$$
\text { (h) } \phi_{i j}=(h) \phi_{i 1} \text {. }
$$

(b) The $\left(y_{i}, h \circ x_{j}\right)$-entries of $L(G, \circ, H)$ and $L^{*}$ are equal if and only if

$$
(h) \phi_{i j}=(h) \phi_{1 j} .
$$

This follows immediately from the facts that $(e) \phi_{i 1}=(e) \phi_{1 j}=e$.

The emphasis of this section is on exploring the relationships between the bijections $\phi_{i 1}, \phi_{1 j}$ and $\phi_{i j}$. So, at this point, it is worth examining an example which highlights various aspects of these relations. In order to display information about several subgroups on one table we shall not restrict ourselves to a canonical headline and sideline. Instead, we choose a headline that exhibits the coset decomposition with respect to the various subgroups. Also, since subgroups in question are normal, we take the sideline to be equal to the headline.

Let $G=\left\{e, a^{4}, a, a^{5}, a^{2}, a^{6}, a^{3}, a^{7}, b, a^{4} b, a b, a^{5} b, a^{2} b, a^{6} b, a^{3} b, a^{7} b\right\}$ and take the following two groups of order 16 :

$$
\begin{aligned}
& (G, \circ) \quad \text { where } \quad a^{8}=e, \quad b^{2}=e, \quad b a=a^{-1} b \quad \text { (the dihedral group), } \\
& (G, *) \quad \text { where } \quad a^{8}=e, \quad b^{2}=e, \quad b a=a^{5} b .
\end{aligned}
$$

Using the ordering given above the multiplication table for the first group (the dihedral group) is: 


\begin{tabular}{|c|c|c|c|c|c|c|c|c|c|c|c|c|c|c|c|c|}
\hline & $e$ & $a^{4}$ & $a$ & $a^{5}$ & $a^{2}$ & $a^{6}$ & $a^{3}$ & $a^{7}$ & $b$ & $a^{4} b$ & $a b$ & $a^{5} b$ & $a^{2} b$ & $a^{6} b$ & $a^{3} b$ & $a^{7} b$ \\
\hline$e$ & $e$ & $a^{4}$ & $a$ & $a^{5}$ & $a^{2}$ & $a^{6}$ & $a^{3}$ & $a^{7}$ & $b$ & $a^{4} b$ & $a b$ & $a^{5} b$ & $a^{2} b$ & $a^{6} b$ & $a^{3} b$ & $a^{7} b$ \\
\hline$a^{4}$ & $a^{4}$ & $e$ & $a^{5}$ & $a$ & $a^{6}$ & $a^{2}$ & $a^{7}$ & $a^{3}$ & $a^{4} b$ & $b$ & $a^{5} b$ & $a b$ & $a^{6} b$ & $a^{2} b$ & $a^{7} b$ & $a^{3} b$ \\
\hline$a$ & $a$ & $a^{5}$ & $a^{2}$ & $a^{6}$ & $a^{3}$ & $a^{7}$ & $a^{4}$ & $e$ & $a b$ & $a^{5} b$ & $a^{2} b$ & $a^{6} b$ & $a^{3} b$ & $a^{7} b$ & $a^{4} b$ & $b$ \\
\hline$a^{5}$ & $a^{5}$ & $a$ & $a^{6}$ & $a^{2}$ & $a^{7}$ & $a^{3}$ & $e$ & $a^{4}$ & $a^{5} b$ & $a b$ & $a^{6} b$ & $a^{2} b$ & $a^{7} b$ & $a^{3} b$ & $a^{4} b$ & $b$ \\
\hline$a^{2}$ & $a^{2}$ & $a^{6}$ & $a^{3}$ & $a^{7}$ & $a^{4}$ & $e$ & $a^{5}$ & $a$ & $a^{2} b$ & $a^{6} b$ & $a^{3} b$ & $a^{7} b$ & $b$ & $a^{4} b$ & $a^{5} b$ & $a b$ \\
\hline$a^{6}$ & $a^{6}$ & $a^{2}$ & $a^{7}$ & $a^{3}$ & $e$ & $a^{4}$ & $a$ & $a^{5}$ & $a^{6} b$ & $a^{2} b$ & $a^{7} b$ & $a^{3} b$ & $b$ & $a^{4} b$ & $a b$ & $a^{5} b$ \\
\hline$a^{3}$ & $a^{3}$ & $a^{7}$ & $a^{4}$ & $e$ & $a^{5}$ & $a$ & $a^{6}$ & $a^{2}$ & $a^{3} b$ & $a^{7} b$ & $a^{4} b$ & $b$ & $a^{5} b$ & $a b$ & $a^{6} b$ & $a^{2} b$ \\
\hline$a^{7}$ & $a^{7}$ & $a^{3}$ & $e$ & $a^{4}$ & $a$ & $a^{5}$ & $a^{2}$ & $a^{6}$ & $a^{7} b$ & $a^{3} b$ & $b$ & $a^{4} b$ & $a b$ & $a^{5} b$ & $a^{2} b$ & $a^{6} b$ \\
\hline$b$ & $b$ & $a^{4} b$ & $a^{7} b$ & $a^{3} b$ & $a^{6} b$ & $a^{2} b$ & $a^{5} b$ & $a b$ & $e$ & $a^{4}$ & $a^{7}$ & $a^{3}$ & $a^{6}$ & $a^{2}$ & $a^{5}$ & $a$ \\
\hline$a^{4} b$ & $a^{4} b$ & $b$ & $a^{3} b$ & $a^{7} b$ & $a^{2} b$ & $a^{6} b$ & $a b$ & $a^{5} b$ & $a^{4}$ & $e$ & $a^{3}$ & $a^{7}$ & $a^{2}$ & $a^{6}$ & $a$ & $a^{5}$ \\
\hline$a b$ & $a b$ & $a^{5} b$ & $b$ & $a^{4} b$ & $a^{7} b$ & $a^{3} b$ & $a^{6} b$ & $a^{2} b$ & $a$ & $a^{5}$ & $e$ & $a^{4}$ & $a^{7}$ & $a^{3}$ & $a^{6}$ & $a^{2}$ \\
\hline$a^{5} b$ & $a^{5} b$ & $a b$ & $a^{4} b$ & $b$ & $a^{3} b$ & $a^{7} b$ & $a^{2} b$ & $a^{6} b$ & $a^{5}$ & $a$ & $a^{4}$ & $e$ & $a^{3}$ & $a^{7}$ & $a^{2}$ & $a^{6}$ \\
\hline$a^{2} b$ & $a^{2} b$ & $a^{6} b$ & $a b$ & $a^{5} b$ & $b$ & $a^{4} b$ & $a^{7} b$ & $a^{3} b$ & $a^{2}$ & $a^{6}$ & $a$ & $a^{5}$ & $e$ & $a^{4}$ & $a^{7}$ & $a^{3}$ \\
\hline$a^{6} b$ & $a^{6} b$ & $a^{2} b$ & $a^{5} b$ & $a b$ & $a^{4} b$ & $b$ & $a^{3} b$ & $a^{7} b$ & $a^{6}$ & $a^{2}$ & $a^{5}$ & $a$ & $a^{4}$ & $e$ & $a^{3}$ & $a^{7}$ \\
\hline$a^{3} b$ & $a^{3} b$ & $a^{7} b$ & $a^{2} b$ & $a^{6} b$ & $a b$ & $a^{5} b$ & $b$ & $a^{4} b$ & $a^{3}$ & $a^{7}$ & $a^{2}$ & $a^{6}$ & $a$ & $a^{5}$ & $e$ & $a^{4}$ \\
\hline$a^{7} b$ & $a^{7} b$ & $a^{3} b$ & $a^{6} b$ & $a^{2} b$ & $a^{5} b$ & $a b$ & $a^{4} b$ & $b$ & $a^{7}$ & $a^{3}$ & $a^{6}$ & $a^{2}$ & $a^{5}$ & $a$ & $a^{4}$ & $e$ \\
\hline
\end{tabular}

Using the same ordering the multiplication table for the second group is: 


\begin{tabular}{|c|c|c|c|c|c|c|c|c|c|c|c|c|c|c|c|c|}
\hline & $e$ & $a^{4}$ & $a$ & $a^{5}$ & $a^{2}$ & $a^{6}$ & $a^{3}$ & $a^{7}$ & $b$ & $a^{4} b$ & $a b$ & $a^{5} b$ & $a^{2} b$ & $a^{6} b$ & $a^{3} b$ & $a^{7} b$ \\
\hline$e$ & $e$ & $a^{4}$ & $a$ & $a^{5}$ & $a^{2}$ & $a^{6}$ & $a^{3}$ & $a^{7}$ & $b$ & $a^{4} b$ & $a b$ & $a^{5} b$ & $a^{2} b$ & $a^{6} b$ & $a^{3} b$ & $a^{7} b$ \\
\hline$a^{4}$ & $a^{4}$ & $e$ & $a^{5}$ & $a$ & $a^{6}$ & $a^{2}$ & $a^{7}$ & $a^{3}$ & $a^{4} b$ & $b$ & $a^{5} b$ & $a b$ & $a^{6} b$ & $a^{2} b$ & $a^{7} b$ & $a^{3} b$ \\
\hline$a$ & $a$ & $a^{5}$ & $a^{2}$ & $a^{6}$ & $a^{3}$ & $a^{7}$ & $a^{4}$ & $e$ & $a b$ & $a^{5} b$ & $a^{2} b$ & $a^{6} b$ & $a^{3} b$ & $a^{7} b$ & $a^{4} b$ & $b$ \\
\hline$a^{5}$ & $a^{5}$ & $a$ & $a^{6}$ & $a^{2}$ & $a^{7}$ & $a^{3}$ & $e$ & $a^{4}$ & $a^{5} b$ & $a b$ & $a^{6} b$ & $a^{2} b$ & $a^{7} b$ & $a^{3} b$ & $a^{4} b$ & $b$ \\
\hline$a^{2}$ & $a^{2}$ & $a^{6}$ & $a^{3}$ & $a^{7}$ & $a^{4}$ & $e$ & $a^{5}$ & $a$ & $a^{2} b$ & $a^{6} b$ & $a^{3} b$ & $a^{7} b$ & $b$ & $a^{4} b$ & $a^{5} b$ & $a b$ \\
\hline$a^{6}$ & $a^{6}$ & $a^{2}$ & $a^{7}$ & $a^{3}$ & $e$ & $a^{4}$ & $a$ & $a^{5}$ & $a^{6} b$ & $a^{2} b$ & $a^{7} b$ & $a^{3} b$ & $b$ & $a^{4} b$ & $a b$ & $a^{5} b$ \\
\hline$a^{3}$ & $a^{3}$ & $a^{7}$ & $a^{4}$ & $e$ & $a^{5}$ & $a$ & $a^{6}$ & $a^{2}$ & $a^{3} b$ & $a^{7} b$ & $a^{4} b$ & $b$ & $a^{5} b$ & $a b$ & $a^{6} b$ & $a^{2} b$ \\
\hline$a^{7}$ & $a^{7}$ & $a^{3}$ & $e$ & $a^{4}$ & $a$ & $a^{5}$ & $a^{2}$ & $a^{6}$ & $a^{7} b$ & $a^{3} b$ & $b$ & $a^{4} b$ & $a b$ & $a^{5} b$ & $a^{2} b$ & $a^{6} b$ \\
\hline$b$ & $b$ & $a^{4} b$ & $a^{5} b$ & $a b$ & $a^{2} b$ & $a^{6} b$ & $a^{7} b$ & $a^{3} b$ & $e$ & $a^{4}$ & $a^{5}$ & $a$ & $a^{2}$ & $a^{6}$ & $a^{7}$ & $a^{3}$ \\
\hline$a^{4} b$ & $a^{4} b$ & $b$ & $a b$ & $a^{5} b$ & $a^{6} b$ & $a^{2} b$ & $a^{3} b$ & $a^{7} b$ & $a^{4}$ & $e$ & $a$ & $a^{5}$ & $a^{6}$ & $a^{2}$ & $a^{3}$ & $a^{7}$ \\
\hline$a b$ & $a b$ & $a^{5} b$ & $a^{6} b$ & $a^{2} b$ & $a^{3} b$ & $a^{7} b$ & $b$ & $a^{4} b$ & $a$ & $a^{5}$ & $a^{6}$ & $a^{2}$ & $a^{3}$ & $a^{7}$ & $e$ & $a^{4}$ \\
\hline$a^{5} b$ & $a^{5} b$ & $a b$ & $a^{2} b$ & $a^{6} b$ & $a^{7} b$ & $a^{3} b$ & $a^{4} b$ & $b$ & $a^{5}$ & $a$ & $a^{2}$ & $a^{6}$ & $a^{7}$ & $a^{3}$ & $a^{4}$ & $e$ \\
\hline$a^{2} b$ & $a^{2} b$ & $a^{6} b$ & $a^{7} b$ & $a^{3} b$ & $a^{4} b$ & $b$ & $a b$ & $a^{5} b$ & $a^{2}$ & $a^{6}$ & $a^{7}$ & $a^{3}$ & $a^{4}$ & $e$ & $a$ & $a^{5}$ \\
\hline$a^{6} b$ & $a^{6} b$ & $a^{2} b$ & $a^{3} b$ & $a^{7} b$ & $b$ & $a^{4} b$ & $a^{5} b$ & $a b$ & $a^{6}$ & $a^{2}$ & $a^{3}$ & $a^{7}$ & $e$ & $a^{4}$ & $a^{5}$ & $a$ \\
\hline$a^{3} b$ & $a^{3} b$ & $a^{7} b$ & $b$ & $a^{4} b$ & $a^{5} b$ & $a b$ & $a^{2} b$ & $a^{6} b$ & $a^{3}$ & $a^{7}$ & $e$ & $a^{4}$ & $a^{5}$ & $a$ & $a^{2}$ & $a^{6}$ \\
\hline$a^{7} b$ & $a^{7} b$ & $a^{3} b$ & $a^{4} b$ & $b$ & $a b$ & $a^{5} b$ & $a^{6} b$ & $a^{2} b$ & $a^{7}$ & $a^{3}$ & $a^{4}$ & $e$ & $a$ & $a^{5}$ & $a^{6}$ & $a^{2}$ \\
\hline
\end{tabular}

Note that the multiplication tables for these groups have a 2 -sided consistent coset decomposition, with respect to the subgroup $H_{1}=\left\{e, a, a^{2}, a^{3}, a^{4}, a^{5}, a^{6}, a^{7}\right\}$, and $\phi_{22}$ is defined. In addition the bijection $\phi_{21}$ is the identity. However neither $\phi_{12}$ nor $\phi_{22}$ is the identity.

The two multiplication tables also have a 2-sided consistent coset decomposition, 
with respect to the subgroup $H_{2}=\left\{e, a^{2}, a^{4}, a^{6}\right\}$, for which all $\phi_{i j}$ are defined. We note that if we let $x_{j}=y_{i}=a$ then both $\phi_{1 j}$ and $\phi_{i 1}$, are the identity, as is $\phi_{i j}$. However we note that if we let $x_{j}=y_{i}=b$ then $\phi_{1 j}$, is the identity but neither $\phi_{i 1}$ nor $\phi_{i j}$ is the identity.

One can also consider the subgroup $H_{3}=\left\{e, a^{4}\right\}$. However it should be noted that in this case the multiplication tables have a 2 -sided consistent coset decomposition, but we do not have $y_{i} \circ H_{3} \circ x_{j}=y_{i} * H_{3} * x_{j}$ for all $i, j$, and hence not all $\phi_{i j}$ are defined. It can be seen that if $y_{i} \in\left\{a, a^{2}, a^{3}\right\}$ and $x_{j}=b$ then $\phi_{1 j}, \phi_{i 1}$, and $\phi_{i j}$ are all equal to the identity.

However, even though $\phi_{i j}$ is defined when $y_{i}=x_{j}=a^{2} b$ and $\phi_{i 1}$ and $\phi_{j 1}$ are the identity, $\phi_{i j}$ is not.

The next result goes some way towards clarifying these relationships.

THEOREM 3.3 Let $1 \leq i, j \leq r$, and if both $i$ and $j$ are greater than 1 , assume $\phi_{i j}$ is defined. Then the Latin subsquare $y_{i} \circ L(H) \circ x_{j}$ of $L(G, \circ, H)$ is equal to the corresponding subsquare of $L^{*}$ if and only if

$$
\phi_{i j}=\phi_{i 1}=\phi_{1 j}
$$

and $\phi_{i j}$ is an isomorphism from $(H, \circ)$ to $(H, *)$. In particular, if this is the case and $i=1$ or $j=1$, then $\phi_{i j}$ is the identity map.

Proof. Suppose first that the subsquare $y_{i} \circ L(H) \circ x_{j}$ of $L(G, \circ, H)$ is equal to the corresponding subsquare of $L^{*}$. By Corollary 3.2 we have $(h) \phi_{i j}=(h) \phi_{1 j}=(h) \phi_{i 1}$ for all $h \in H$. Hence $\phi_{i j}=\phi_{i 1}=\phi_{1 j}$, and so by Proposition 3.1, $\left(h \circ h^{\prime}\right) \phi_{i j}=$ 
$(h) \phi_{i j} *\left(h^{\prime}\right) \phi_{i j}$ for all $h, h^{\prime} \in H$. Since $\phi_{i j}$ is a bijection, it follows that $\phi_{i j}$ is an isomorphism from $(H, \circ)$ to $(H, *)$.

Conversely, suppose that $\phi_{i j}=\phi_{i 1}=\phi_{1 j}$ is an isomorphism from $(H, \circ)$ to $(H, *)$. Then, for $h, h^{\prime} \in H$, the $\left(y_{i} \circ h, h^{\prime} \circ x_{j}\right)$-entry of $L(G, \circ, H)$ is $\left(y_{i} \circ h\right) \circ\left(h^{\prime} \circ x_{j}\right)=$ $y_{i} \circ\left(h \circ h^{\prime}\right) \circ x_{j}$, which by definition of $\phi_{i j}$ is equal to $y_{i} *\left(h \circ h^{\prime}\right) \phi_{i j} * x_{j}$. Also the $\left(y_{i} \circ h, h^{\prime} \circ x_{j}\right)$-entry of $L^{*}$ is $\left(y_{i} \circ h\right) *\left(h^{\prime} \circ x_{j}\right)=\left(y_{i} *(h) \phi_{i 1}\right) *\left(\left(h^{\prime}\right) \phi_{1 j} * x_{j}\right)$ by the definition of $\phi_{i 1}$ and $\phi_{1 j}$, and since $\phi_{i j}=\phi_{i 1}=\phi_{1 j}$ is an isomorphism, this is equal to $y_{i} *\left(h \circ h^{\prime}\right) \phi_{i j} * x_{j}$. Hence the subsquare $y_{i} \circ L(H) \circ x_{j}$ of $L(G, \circ, H)$ is equal to the corresponding subsquare of $L^{*}$. Finally the assertion that $\phi_{i j}$ is the identity map if $i=1$ or $j=1$ follows from Lemma 2.2.

We are interested in the number of subsquares at which the multiplication tables of distinct groups $(G, \circ)$ and $(G, *)$ can coincide. (We say that subarrays coincide if they agree entry by entry.) It is difficult to get a precise solution to this problem, but Theorem 3.3 has the following consequence.

COROLLARY 3.4 If the Latin multiplication tables $L(G, \circ, H)$ and $L^{*}$ coincide on all the subsquares $y_{i} \circ L(H) \circ x_{j}$, with $i>1, j>1$, and at least one of the subsquares $y_{i} \circ L(H) \circ x_{j}$ with $i=1$ or $j=1$ then $L(G, \circ, H)=L^{*}$.

Proof. By Lemma 2.2, $(H, \circ)=(H, *)$ and the assumptions of this section hold. By Theorem 3.3 it follows that $\phi_{i j}$ is equal to the identity map for all $i, j$.

We would like to get a better result than this. However it supports our belief that distinct multiplication tables $L(G, \circ, H)$ and $L^{*}$ must differ in a reasonable proportion 
of the subsquares. We also believe that the position of coinciding subsquares is important. We illustrate this with a sample result.

PROPOSITION 3.5 Suppose that for some $i, j, \phi_{i 1}$ and $\phi_{1 j}$ exist and equal the identity map. Then, for $h, h^{\prime} \in H$, the $\left(y_{i} \circ h, h^{\prime} \circ x_{j}\right)$-entries in $L(G, \circ, H)$ and $L^{*}$ are equal if and only if $h \circ h^{\prime}=\left(h \circ h^{\prime}\right) \phi_{i j}$.

Proof. By Proposition 3.1, equality of the $\left(y_{i} \circ h, h^{\prime} \circ x_{j}\right)$-entries is equivalent to $\left(h \circ h^{\prime}\right) \phi_{i j}=(h) \phi_{i 1} *\left(h^{\prime}\right) \phi_{1 j}=h * h^{\prime}$, which is equal to $h \circ h^{\prime}$ since the multiplication tables for $(H, \circ)$ and $(H, *)$ are identical.

\section{$4 \quad$ Some interesting questions}

In this section we list some interesting questions which arose while we were working on this problem. In some cases we provide an answer or a partial answer to the question. We include these as we feel they are of interest to the discussion in general. We close the paper with a curiosity involving the multiplication tables of the dihedral and quaternion groups of order 8. In what follows we assume that we have two groups $(G, \circ)$ and $(G, *)$ and that we are discussing their multiplication tables. We denote the inverse of $g \in G$ in the groups $(G, \circ)$ and $(G, *)$ by $g^{-1}$ and $g^{*}$ respectively. In addition we note that we can recognise the identity elements $e$ and $e_{*}$ from the multiplication tables for $(G, \circ)$ and $(G, *)$ respectively, since we require our multiplication tables to have sidelines and headlines. By permuting the elements of $(G, *)$ we may therefore assume that $e=e_{*}$. While this may not necessarily be what we wish to do for 
comparing two Latin squares, it is a reasonable assumption if we wish to compare the influence of the subgroup structure on the Latin square structure. We begin by investigating what happens when two multiplication tables have a Latin subsquare $S$ in common.

Question 1: What can we say if the two multiplication tables coincide on a subsquare of order 2 ?

Partial Answer: It is obvious that this one condition will not force the multiplication tables to coincide in all entries. However we can deduce some information about subgroups in the corresponding groups.

If we take a group $(G, \circ)$, then it is easy to verify that every subsquare of the multiplication table of $G$ is of the form $y H \circ H x$, for some $H$ a subgroup of $G$. Consequently, if the multiplication tables for two groups coincide in a subsquare $S$ of order 2, there must exist subgroups $H$ and $H^{\prime}$ of order 2 in the groups such that $S$ is a subsquare in the respective coset decompositions.

Formalising these ideas we obtain:

PROPOSITION 4.1 (a) Let $c \in G$. Then the multiplication table for $(G, \circ)$ has a $2 \times 2$-subsquare $S$ of the form

$\begin{array}{ll}e & x \\ x & e\end{array}$

with one of the rows of $S$ corresponding to row $c$ of the multiplication table for $(G, \circ)$ if and only if $x$ has order 2 in $(G, \circ)$, and setting $H=<c^{-1} \circ x \circ c>=\left\{c^{-1} \circ x \circ c, e\right\}=$ 
$\{y, e\}, S$ forms the subsquare $c \circ L(H) \circ c^{-1}$ in the canonical coset decomposition of $L(G, \circ, H)$ relative to $H$.

(b) Now suppose that $e=e_{*}$. Then the multiplication tables for $(G, \circ)$ and $(G, *)$ have the subsquare $S$ above in common, where $S$ involves row $c$, if and only if

$$
x \circ x=x * x=e, \quad c^{-1}=c^{*}, \quad x \circ c=x * c \quad c^{-1} \circ x=c^{*} * x .
$$

Moreover, if this is the case, then $S=c \circ L(H) \circ c^{-1}=c * L\left(H^{\prime}\right) * c^{*}$, where $H:=\{y, e\}$ and $H^{\prime}:=\left\{y^{\prime}, e\right\}$ with $y:=c^{-1} \circ x \circ c$ and $y^{\prime}:=c^{*} * x * c$ of order 2 in $(G, \circ)$ and $(G, *)$ respectively; and $y=y^{\prime}$ if and only if $c^{*} *(x * c)=c^{*} \circ(x * c)$.

Proof. (a) Suppose first that such an $S$ is a subsquare. Then it is immediate from the above remark that $x \circ x=e$. Let the rows involved in $S$ correspond to elements $c$ and $d$ of $G$. Then, considering the diagonal entries of $S$, the columns involved in $S$ must correspond to $c^{-1}$ and $d^{-1}$. Now $d \circ c^{-1}=x$ implies that $d=x \circ c=c \circ y$, and $d^{-1}=c^{-1} \circ x=y \circ c^{-1}$. Hence $S=c \circ L(H) \circ c^{-1}$ with $H$ as in part (a). Conversely, if $y$ has order 2 in $(G, \circ)$, then, for any $c \in G$, setting $x:=c \circ y \circ c^{-1}$ and $H:=<y>=\{y, e\}$, we have $c \circ L(H) \circ c^{-1}$ equal to:

$\begin{array}{ll}e & x \\ x & e\end{array}$

(b) Once again by the remark above, if $S$ is in common, then $x \circ x=x * x=$ $e, \quad c^{-1}=c^{*}, x \circ c=x * c$ and $c^{-1} \circ x=c^{*} * x$. Conversely, let $x$ have order 2 in both groups, and let $c$ be such that $c^{-1}=c^{*}$ and $x \circ c=x * c=d$ say and $c^{-1} \circ x=c^{*} * x$. Then $d^{-1}=c^{-1} \circ x=c^{*} * x=d^{*}$ and the subsquare $S$ corresponding to rows $c$ and $d$ and columns $c^{-1}$ and $d^{-1}=d^{*}$ is 


$\begin{array}{ll}e & x \\ x & e .\end{array}$

Moreover, defining $y, y^{\prime}$ as in part (b), it is easily checked that $S=c \circ L(H) \circ c^{-1}=$ $c * L\left(H^{\prime}\right) * c^{*}$ and $y=y^{\prime}$ if and only if $c^{*} *(x * c)=c^{*} \circ(x * c)$.

The subgroups $H$ and $H^{\prime}$, of the groups $(G, \circ)$ and $(G, *)$ respectively, may not be the same, but each is a conjugate (in the relevant group) of the common subgroup $\{x, e\}$. The rows involved in $S$ are row $c$ and row $d:=x \circ c=x * c$, and the columns are column $c^{-1}=c^{*}$ and column $d^{-1}=d^{*}=c^{-1} \circ x=c^{*} * x$.

We now examine the column $c^{-1}$ to see where the entries $c, d$ occur in the two multiplication tables. In $L(G, \circ, H)$ they will be in rows $c \circ c$ and $x \circ(c \circ c)$ respectively, while in $L^{*}$ they will be in rows $c * c$ and $x * c * c$ respectively. If we assume in addition that these two entries coincide in the two multiplication tables then we will have that

$$
c * c=c \circ c, \quad x \circ c \circ c=x * c * c
$$

Now we investigate the situation in which the multiplication tables coincide in a specified set of subsquares in a 2 -sided consistent coset decomposition induced by the $\operatorname{subgroups}(H, \circ)$ and $(H, *)$.

Question 2: If the multiplication tables $L(G, \circ, H)$ and $L^{*}$ coincide in all subsquares of the form $y_{i} \circ L(H)$ and all subsquares of the form $L(H) \circ x_{i}$, as well as in a transversal of subsquares, then are $L(G, \circ, H)$ and $L^{*}$ identical?

Partial Answer: This condition does not imply that the multiplication tables will agree everywhere. 
Below we give the multiplication table for a group of order 16. If this multiplication table is compared with the multiplication table for the dihedral group given in Section 3 , it can be seen that the two multiplication tables agree in all subsquares in the first row and first column, and in addition in all the subsquares in some transversal of subsquares. (Note that for purposes of comparison we have used the same headline and sideline as were used in the example in Section 3, rather than the canonical one.)

Let $G=\left\{e, a^{4}, a, a^{5}, a^{2}, a^{6}, a^{3}, a^{7}, b, a^{4} b, a b, a^{5} b, a^{2} b, a^{6} b, a^{3} b, a^{7} b\right\}$ and take the following group:

$$
(G, *) \quad \text { where } \quad a^{8}=e, \quad b^{2}=e, \quad b a=a^{3} b
$$

Use the ordering given in the set above to label the headline and sideline of the multiplication table.

The multiplication table for this group is: 


\begin{tabular}{|c|c|c|c|c|c|c|c|c|c|c|c|c|c|c|c|c|}
\hline & $e$ & $a^{4}$ & $a$ & $a^{5}$ & $a^{2}$ & $a^{6}$ & $a^{3}$ & $a^{7}$ & $b$ & $a^{4} b$ & $a b$ & $a^{5} b$ & $a^{2} b$ & $a^{6} b$ & $a^{3} b$ & $a^{7} b$ \\
\hline$e$ & $e$ & $a^{4}$ & $a$ & $a^{5}$ & $a^{2}$ & $a^{6}$ & $a^{3}$ & $a^{7}$ & $b$ & $a^{4} b$ & $a b$ & $a^{5} b$ & $a^{2} b$ & $a^{6} b$ & $a^{3} b$ & $a^{7} b$ \\
\hline$a^{4}$ & $a^{4}$ & $e$ & $a^{5}$ & $a$ & $a^{6}$ & $a^{2}$ & $a^{7}$ & $a^{3}$ & $a^{4} b$ & $b$ & $a^{5} b$ & $a b$ & $a^{6} b$ & $a^{2} b$ & $a^{7} b$ & $a^{3} b$ \\
\hline$a$ & $a$ & $a^{5}$ & $a^{2}$ & $a^{6}$ & $a^{3}$ & $a^{7}$ & \begin{tabular}{|l|}
$a^{4}$ \\
\end{tabular} & $e$ & $a b$ & $a^{5} b$ & $a^{2} b$ & $a^{6} b$ & $a^{3} b$ & $a^{7} b$ & $a^{4} b$ & $b$ \\
\hline$a^{5}$ & $a^{5}$ & $a$ & $a^{6}$ & $a^{2}$ & $a^{7}$ & $a^{3}$ & $e$ & \begin{tabular}{|l|}
$a^{4}$ \\
\end{tabular} & $a^{5} b$ & $a b$ & $a^{6} b$ & $a^{2} b$ & $a^{7} b$ & $a^{3} b$ & $b$ & $a^{4} b$ \\
\hline$a^{2}$ & $a^{2}$ & $a^{6}$ & $a^{3}$ & $a^{7}$ & $a^{4}$ & $e$ & $a^{5}$ & $a$ & $a^{2} b$ & $a^{6} b$ & $a^{3} b$ & $a^{7} b$ & $a^{4} b$ & $b$ & $a^{5} b$ & $a b$ \\
\hline$a^{6}$ & $a^{6}$ & $a^{2}$ & $a^{7}$ & $a^{3}$ & $e$ & $a^{4}$ & $a$ & $a^{5}$ & $a^{6} b$ & $a^{2} b$ & $a^{7} b$ & $a^{3} b$ & $b$ & $a^{4} b$ & $a b$ & $a^{5} b$ \\
\hline$a^{3}$ & $a^{3}$ & $a^{7}$ & $a^{4}$ & $e$ & $a^{5}$ & $a$ & $a^{6}$ & $a^{2}$ & $a^{3} b$ & $a^{7} b$ & $a^{4} b$ & $b$ & $a^{5} b$ & $a b$ & $a^{6} b$ & $a^{2} b$ \\
\hline$a^{7}$ & $a^{7}$ & $a^{3}$ & $e$ & $a^{4}$ & $a$ & $a^{5}$ & $a^{2}$ & $a^{6}$ & $a^{7} b$ & $a^{3} b$ & $b$ & $a^{4} b$ & $a b$ & $a^{5} b$ & $a^{2} b$ & $a^{6} b$ \\
\hline$b$ & $b$ & $a^{4} b$ & $a^{3} b$ & $a^{7} b$ & $a^{6} b$ & $a^{2} b$ & $a b$ & $a^{5} b$ & $e$ & $a^{4}$ & $a^{3}$ & $a^{7}$ & \begin{tabular}{|l|}
$a^{6}$ \\
\end{tabular} & $a^{2}$ & $a$ & $a^{5}$ \\
\hline$a^{4} b$ & $a^{4} b$ & $b$ & $a^{7} b$ & $a^{3} b$ & $a^{2} b$ & $a^{6} b$ & $a^{5} b$ & $a b$ & $a^{4}$ & $e$ & $a^{7}$ & $a^{3}$ & $a^{2}$ & $a^{6}$ & $a^{5}$ & $a$ \\
\hline$a b$ & $a b$ & $a^{5} b$ & $a^{4} b$ & $b$ & $a^{7} b$ & $a^{3} b$ & $a^{2} b$ & $a^{6} b$ & $a$ & $a^{5}$ & $a^{4}$ & $e$ & $a^{7}$ & $a^{3}$ & $a^{2}$ & $a^{6}$ \\
\hline$a^{5} b$ & $a^{5} b$ & $a b$ & $b$ & $a^{4} b$ & $a^{3} b$ & $a^{7} b$ & $a^{6} b$ & $a^{2} b$ & $a^{5}$ & $a$ & $e$ & $a^{4}$ & $a^{3}$ & $a^{7}$ & $a^{6}$ & $a^{2}$ \\
\hline$a^{2} b$ & $a^{2} b$ & $a^{6} b$ & $a^{5} b$ & $a b$ & $b$ & $a^{4} b$ & $a^{3} b$ & $a^{7} b$ & $a^{2}$ & $a^{6}$ & $a^{5}$ & $a$ & $e$ & $a^{4}$ & $a^{3}$ & $a^{7}$ \\
\hline$a^{6} b$ & $a^{6} b$ & $a^{2} b$ & $a b$ & $a^{5} b$ & $a^{4} b$ & $b$ & $a^{7} b$ & $a^{3} b$ & $a^{6}$ & $a^{2}$ & $a$ & $a^{5}$ & $a^{4}$ & $e$ & $a^{7}$ & $a^{3}$ \\
\hline$a^{3} b$ & $a^{3} b$ & $a^{7} b$ & $a^{6} b$ & $a^{2} b$ & $a b$ & $a^{5} b$ & $a^{4} b$ & $b$ & $a^{3}$ & $a^{7}$ & $a^{6}$ & $a^{2}$ & $a$ & $a^{5}$ & $a^{4}$ & $e$ \\
\hline$a^{7} b$ & $a^{7} b$ & $a^{3} b$ & $a^{2} b$ & $a^{6} b$ & $a^{5} b$ & $a b$ & $b$ & $a^{4} b$ & $a^{7}$ & $a^{3}$ & $a^{2}$ & $a^{6}$ & $a^{5}$ & $a$ & $e$ & $a^{4}$ \\
\hline
\end{tabular}

It can be seen that $H=\left\{e, a^{4}\right\}$ is a subgroup of both this group and the dihedral group. In addition the boxed entries form a transversal of subsquares. Further the two multiplication tables agree in the first two rows and columns and in the transversal of subsquares, but are not identical.

What if we increase the proportions of subsquares which must coincide? 
Question 3: In how large a proportion of subsquares can $L(G, \circ, H)$ and $L^{*}$ coincide without being equal?

Partial Answer: It can be seen that the multiplication tables given above and the multiplication table for the dihedral group given in Section 3 agree in $75 \%$ of all subsquares of the form $y_{i} L(H) x_{j}$, where $H=\left\{e, a^{4}\right\}$ and $1 \leq i, j \leq 8$, and yet still do not agree everywhere.

For a more general example we may take $n \geq 4$ and consider the groups, of order $2^{n}$, with generators $a$ and $b$, and determined by the relations

(1) $a^{2^{n-1}}=e, b^{2}=a^{2^{n-2}}, b a=a^{-1} b$, the generalized quaternion group,

(2) $a^{2^{n-1}}=e, b^{2}=e, b a=a^{-1} b$, the dihedral group,

(3) $a^{2^{n-1}}=e, b^{2}=e, b a=a^{1+2^{n-2}} b$,

(4) $a^{2^{n-1}}=e, b^{2}=e, b a=a^{-1+2^{n-2}} b$.

If we fix $n$ and use the cosets $H=\left\{e, a^{2^{n-2}}\right\}, a H, a^{2} H, \ldots, a^{2^{n-2}-1} H, b H, a b H, a^{2} b H$, $\ldots, a^{2^{n-2}-1} b H$ to label the headline and sideline of the multiplication tables for these groups, then we obtain examples of groups which agree in $75 \%$ of all subsquares, of the form $y_{i} L(H) x_{j}$, where $H=\left\{e, a^{4}\right\}$ and $1 \leq i, j \leq 2^{n-1}$, but not everywhere.

It is easy to see that given this labelling the multiplication tables of these four groups will agree in all rows indexed by $H=\left\{e, a^{2^{n-2}}\right\}, a H, a^{2} H, \ldots, a^{2^{n-2}-1} H$ and so they must agree in $50 \%$ of all such subsquares. However we want more.

LEMMA 4.2 The multiplication tables of the dihedral group of order $2^{n}$ with gen- 
erating relations

$$
a^{2^{n-1}}=e, b^{2}=e, b a=a^{-1} b
$$

and the group of order $2^{n}$ with generating relations

$$
a^{2^{n-1}}=e, b^{2}=e, b a=a^{-1+2^{n-2}} b
$$

agree in $75 \%$ of all subsquares of the form $y_{i} L(H) x_{j}$, where $H=\left\{e, a^{2^{n-2}}\right\}$ and $1 \leq i, j \leq 2^{n-1}$

Proof First consider the dihedral group. Here

$$
a^{r} b a^{s}=a^{r-s} b
$$

and in the second group

$$
a^{r} b a^{s}=a^{r-s+s 2^{n-2}} b
$$

If $s$ is even, then $a^{s 2^{n-2}}=\left(a^{2^{n-1}}\right)^{s / 2}=e$ and the multiplication tables will coincide in entries of the form $a^{r} b a^{s}$. Observe that in the dihedral group

$$
a^{r} b a^{s} b=a^{r-s} b^{2}=a^{r-s}
$$

and in the second group

$$
a^{r} b a^{s} b=a^{r-s+s 2^{n-2}} b^{2}=a^{r-s+s 2^{n-2}}
$$

and so the two multiplication tables will coincide when $s$ is even and the result follows.

In a similar fashion, we can calculate the percentage of subsquares of the form $y_{i} L(H) x_{j}$ in which the various multiplication tables of all four groups of order $2^{n}$ given above agree. (Here, again, $H$ is the subgroup $\left\{e, a^{2^{n-2}}\right\}$ of order 2.) The results are shown in the table below. 


\begin{tabular}{l|ccccc}
$(G, \circ)$ & 1 & 1 & 1 & 2 & 3 \\
$(G, *)$ & 2 & 4 & 3 & 3 & 4 \\
\% equal subsquares & 75 & 75 & $<75$ & $<75$ & $<75$
\end{tabular}

In fact, as was pointed out by one of the referees, if we take any two groups whose multiplication tables agree in $75 \%$ of subsquares, then the direct products of these groups with the cyclic group $C_{r}$ have the same properties, providing an infinite set of examples.

The subgroup $H=\left\{e, a^{2^{n-2}}\right\}$ of order 2 used in the above example is normal, and it seems reasonable to investigate the following question.

Question 4: If $(H, \circ)=(H, *)$, where $(H, \circ)$ is a normal subgroup in $(G, \circ)$ and $(H, *)$ is a subgroup in $(G, *)$, under what conditions on the multiplication tables $L(G, \circ, H)$ and $L(G, *, H)$ can we deduce that $(H, *)$ is normal in $(G, *) ?$

Partial Answer: Note that for normality of $(H, *)$ in $(G, *)$, it is not sufficient simply to assume that $(H, \circ)=(H, *)$, as is evident on considering any two groups $G_{1}$ and $G_{2}$ of the same order with isomorphic subgroups $H_{1}, H_{2}$ of $G_{1}, G_{2}$ respectively such that $H_{1}$ is normal in $G_{1}$, but $H_{2}$ is not normal in $G_{2}$, (for example $Z_{6}$ and $S_{3}$ with subgroups of order 2). However, to assume that $(G, \circ)$ and $(G, *)$ have a consistent 2 -sided coset decomposition with respect to $\mathbf{x}=\left(x_{1}, \ldots, x_{r}\right)$ and $\mathbf{y}=\left(y_{1}, \ldots, y_{r}\right)$, is too strong as it is immediate that $(H, *)$ is a normal subgroup of $(G, *)$.

Also it is not clear what can be deduced from the fact that $H$ is abelian and normal. We also lack examples of groups with distinct unit elements whose multiplication 
tables differ in only a small number of places.

We leave the reader with a nice example.

If we rewrite the multiplication tables for the quaternion and dihedral groups of order 8 using $G=\left\{e, a^{-1}, a^{2}, a, b^{-1}, a^{-1} b^{-1}, a^{2} b^{-1}, a b^{-1}\right\}$ and the relations $a^{4}=e, b^{2}=$ $e, b^{-1} a b=a^{-1}$ for the dihedral group and relations $a^{4}=e, b^{-1} a b=a^{-1}, b^{2}=a^{2}$ for the quaternion group then we may write the multiplication tables for both these groups as follows.

\begin{tabular}{c|cccccccc}
$\circ$ & $e$ & $a^{-1}$ & $a^{2}$ & $a$ & $b^{-1}$ & $a^{-1} b^{-1}$ & $a^{2} b^{-1}$ & $a b^{-1}$ \\
\hline$e$ & $e$ & $a^{-1}$ & $a^{2}$ & $a$ & $b^{-1}$ & $a^{-1} b^{-1}$ & $a^{2} b^{-1}$ & $a b^{-1}$ \\
$a$ & $a$ & $e$ & $a^{-1}$ & $a^{2}$ & $a b^{-1}$ & $b^{-1}$ & $a^{-1} b^{-1}$ & $a^{2} b^{-1}$ \\
$a^{2}$ & $a^{2}$ & $a$ & $e$ & $a^{-1}$ & $a^{2} b^{-1}$ & $a b^{-1}$ & $b^{-1}$ & $a^{-1} b^{-1}$ \\
$a^{-1}$ & $a^{-1}$ & $a^{2}$ & $a$ & $e$ & $a^{-1} b^{-1}$ & $a^{2} b^{-1}$ & $a b^{-1}$ & $b^{-1}$ \\
$b$ & $b$ & $b a^{-1}$ & $b a^{2}$ & $b a$ & $e$ & $a$ & $a^{2}$ & $a^{-1}$ \\
$b a$ & $b a$ & $a$ & $b a^{-1}$ & $b a^{2}$ & $a^{-1}$ & $e$ & $a$ & $a^{2}$ \\
$b a^{2}$ & $b a^{2}$ & $b a$ & $b$ & $b a^{-1}$ & $a^{2}$ & $a^{-1}$ & $e$ & $a$ \\
$b a^{-1}$ & $b a^{-1}$ & $b a^{2}$ & $b a$ & $b$ & $a$ & $a^{2}$ & $a^{-1}$ & $e$
\end{tabular}

These tables appear to be the same, however there is actually a deception as in the dihedral group $b^{-1}=b$, whereas this is not the case in the quaternion group. (In connection with this example, see the remarks made by Bedford [1] before his Theorem 1.)

\section{ACKNOWLEDGEMENT}


We wish to thank the referees for their candid comments, which have led us to strengthen our results and improve the presentation of this paper.

\section{References}

[1] David Bedford, Transversals in the Cayley tables of the non-cyclic groups of order 8, European J. Combin., 12, (1991), 455-458.

[2] József Dénes, On a problem of L. Fuchs, Acta Sci. Math. (Szeged), 23, (1962), $237-241$.

[3] A. Drápal, How far apart can the group multiplication tables be?, Europ. J. Combinatorics, 13 (1992), 335-343.

[4] L. Fuchs, Abelian Groups, Akadémiai Kiadó, Budapest, 1958. 